\title{
Reference Period
}

National Cancer Institute

\section{Source}

National Cancer Institute. Reference Period. NCI Thesaurus. Code C49637.

A time frame that is of value for its relationship to a notable event. 